\title{
Ergonomix dentalis
}

Welche Leserin und welcher Leser dieser Fachzeitschrift hat schon einmal aufrecht sitzend in nicht verdrehter Haltung, die Oberarme dicht am Körper, mit aufgestützten, angewinkelten Unterarmen einen 2. Molaren im Oberkiefer bukkal präpariert, dabei die Füße stets flach auf dem Boden gehalten und den Kopf nur leicht gebeugt? Wer diese Vorgaben jeden Tag bei jeder Behandlung hundertprozentig beherzigt, kann diesen Beitrag überspringen. Alle anderen sind eingeladen, mit den folgenden Zeilen auf den aktuellen Stand in puncto „dentale Ergonomie“ zu kommen.

Ergonomische Aspekte haben in der Regel keine Auswirkungen darauf, ob heute eine Präparation gelingt. Nicht jetzt, sondern erst in Jahren oder Jahrzehnten spüren der Zahnarzt oder die Zahnärztin die Folgen einer suboptimalen Haltung. So geben 64\% der Zahnärzte Erkrankungen im Bereich der Wirbelsäule an, und $42 \%$ klagen über haltungsbedingte Kopfschmerzen [1]. Laut Statistik arbeiten 70$75 \%$ der Kollegen unter mehr oder weniger starken Schmerzen, und immerhin 7,55\% werden während ihrer Lebensarbeitszeit berufsunfähig [2]. Dabei sind die orthopädischen Beschwerden vor allem auf die Nacken-, Schulter- und die untere Rückenmuskulatur konzentriert [1]. Gerade weil Defizite sich nicht unmittelbar bemerkbar machen, ist das Wissen um vorbeugende Maßnahmen so wichtig.

Immer mehr Komponenten

sind zu integrieren

Wenn es um die Ergonomie in einer Zahnarztpraxis geht, steht die Behandlungseinheit im Mittelpunkt. Dazu gibt es zunächst eine gute Nachricht: Die Weiterentwicklung erfolgt in diesem Bereich evolutiv. Darum braucht sich niemand zu ängstigen, er habe womöglich soeben eine sprunghafte Verbesserung verpasst.

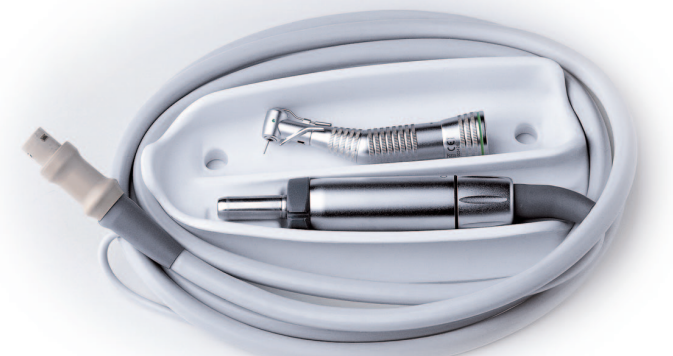

Immer mehr Peripheriegeräte werden von vorneherein in zahnärztliche Behandlungseinheiten integriert - zum Beispiel ein vollsterilisierbarer Mikromotor mit Drehmomentsteuerung für Implantologen.
Auch die erste Behandlungseinheit des Vaters, von dem man die Praxis übernommen hat, wurde in der Regel bereits nach ergonomischen Gesichtspunkten gestaltet.

Eine Veränderung, die sich über die letzten Jahrzehnte vollzogen hat, sticht dennoch unmittelbar ins Auge: Früher hatte man im Wesentlichen die reine Behandlungseinheit, und dazu stellte man im Laufe der Zeit wenige Peripheriegeräte. Deren Anzahl ist heute in der Regel viel größer, und so sollten die chirurgische Einheit, endodontische Geräte, Monitore, Multimedia-Komponenten und vieles mehr gleich in ein ergonomisches Gesamtkonzept integriert sein.

\section{Wichtige Einzelheiten und}

die richtige Mischung

Die Basis sind ein Turbinenanschluss, 2 Motoren, das Zahnsteinentfernungsgerät und die Multifunktionsspritze. Darüber hinaus kommen je nach den PraxisSchwerpunkten weitere Funktionseinheiten hinzu: zum Beispiel für den Implantologen die Kochsalzpumpe und der

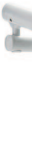

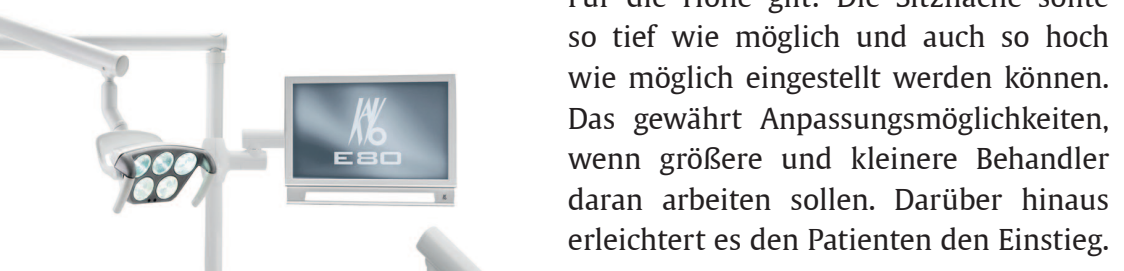

Elektronik braucht Innovation, wie zum Beispiel Beleuchtungssysteme mit einstellbarer Farbtemperatur und „3-D-Achse“.

vollsterilisierbare Mikromotor mit Drehmomentsteuerung. Und nicht zu vergessen die diversen Kameras (Intraoral, Fluoreszenzanalytik, digitales Röntgen)! Moderne Elektronik und Software machen die Integration technisch möglich. Aus ergonomischen Gesichtspunkten ist darauf zu achten, dass der „Kabelsalat“ drumherum nicht stört und die Greifwege zu allen Geräten kurz ausfallen.

Neben der Hand- spielt selbstverständlich auch die Fußarbeit eine Rolle. Bewährt hat sich dafür das SchwebestuhlKonzept mit motorischer Horizontalverschiebung des Patientenstuhls. Diese Kombination gewährt Behandler und Assistenz die maximale Beinfreiheit. Dabei ist das Arztelement seitlich und oberhalb des Patienten angebracht. Optimalerweise wählt man dazu einen funkgesteuerten Fußanlasser. Damit können Kabel komplett vermieden werden.

Für die Höhe gilt: Die Sitzfläche sollte so tief wie möglich und auch so hoch wie möglich eingestellt werden können. Das gewährt Anpassungsmöglichkeiten, wenn größere und kleinere Behandler erleichtert es den Patienten den Einstieg.

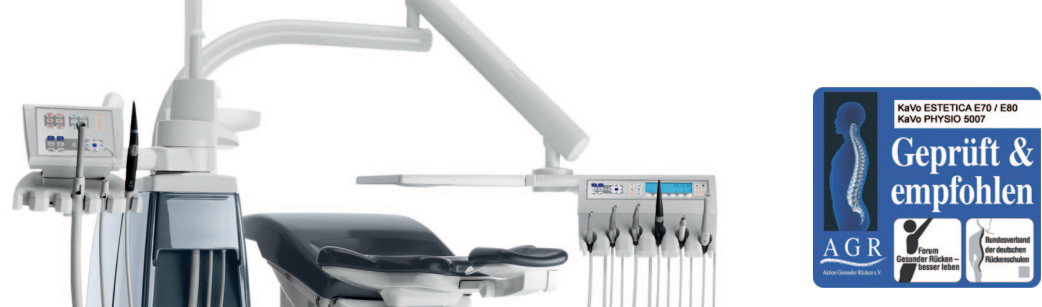

Ausgezeichnet mit dem Siegel der AGR: die KaVo ESTETICA E80 \& E70. 
Selbst bei körperlichen Einschränkungen, wie zum Beispiel allgemein bei Senioren oder speziell bei Hohlkreuz oder Rundrücken, findet sich dafür eine gute Position. Dies ermöglicht die motorische Sitzbankanhebung (z. B. KaVo ESTETICA E80).

Auf ein Detail, das über Design und moderner Technik zuweilen vergessen wird, sollte bei der Anschaffung einer Behandlungseinheit besonders geachtet werden: die Rückenlehne. Kleinere Behandler wählen bevorzugt eine schmale Version; damit wird ein extremes Herüberbeugen über den Patienten vermieden.

Eine unterschätzte ergonomische Bedeutung kommt der richtigen Beleuchtung zu. Die zeitgemäße LED-Lampe sollte mindestens nach vorne und hinten schwenkbar sein, idealerweise aber auch nach links und rechts („3-D-Gelenk“). So lassen sich selbst schwer einsehbare Bereiche gut ausleuchten, auch für die bukkale Präparation des besagten 2. Molaren im Oberkiefer. Beim späteren Legen der Füllung darf die Wellenlänge nicht die Aushärtung des Adhäsivs bzw. Komposits initiieren. Sonst verkürzt sich überraschend die Modellierzeit, was dann zu hektischem Arbeiten und letztlich auch zu ergonomisch ungünstigen Bewegungen führt. Die Farbtemperatur der LEDLampe lässt sich bei modernen Geräten individuell einstellen, auch auf Tageslicht ohne den früher einmal üblichen „Blaustich“. Bei der Bedienung ist aus ergonomischen Gesichtspunkten eine kontaktlose Sensorschaltung am Leuchtenkopf wünschenswert.

Fazit: Linderung braucht Geduld, Vorbeugung Köpfchen

Wer bei sich bereits einen hohen Muskeltonus feststellt (Musculus masseter und Musculus temporalis), sollte einen Physiotherapeuten um Rat fragen. Sowohl die klassische als auch die manuelle Therapie können helfen. Dabei gilt es, mit sich selbst Geduld zu haben! Denn wie die Beschwerden sich über längere Zeit einschleichen, so wird auch die Linderung erst nach Wochen oder Monaten spürbar - dann aber nachhaltig. Beim zusätzlichen Ausgleichssport gilt im Allgemeinen: weniger Tennis, mehr Golf.

Zur Vorbeugung empfiehlt es sich aber insbesondere beim Erwerb einer Behandlungseinheit die vorstehend aufgeführten Punkte zu beachten. Immerhin arbeitet der Zahnarzt bzw. die Zahnärztin im Durchschnitt mindestens $7-8 \mathrm{~h}$ pro Tag daran - bei vielen ist es sogar mehr. Neben der guten Nachricht, dass die Weiterentwicklung evolutiv erfolgt, gibt es übrigens eine 2. positive zu vermelden: Unabhängige Institutionen bewerten inzwischen Zahnarztstühle (z.B. AGR, Aktion Gesunder Rücken e.V.). Dabei gehen viele Aspekte ein, denn gerade hier gilt: Jede Kleinigkeit und der richtige „GesamtMix" machen eine gute Behandlungseinheit aus. Erhält sie bei der externen Begutachtung unterm Strich Top-Noten, sollte das zahnärztliche Team damit ergonomisch sicher im grünen Bereich liegen.

\section{Literatur}

1 Rohmert W., Mainzer J., Zipp P. Der Zahnarzt im Blickfeld der Ergonomie - Eine Analyse zahnärztlicher Arbeitshaltungen (Forschungsinstitut für die zahnärztliche Versorgung). Köln: Deutscher Ärzte-Verlag; 1986

2 Neddermeyer U. Ein besonderes Behandlungskonzept. zm 2011; 101: 38ff.

\section{Korrespondenzadresse}

Marcel Meurer

dNA - dentale Nachrichten-Agentur GmbH m.meurer@d-n-a.eu

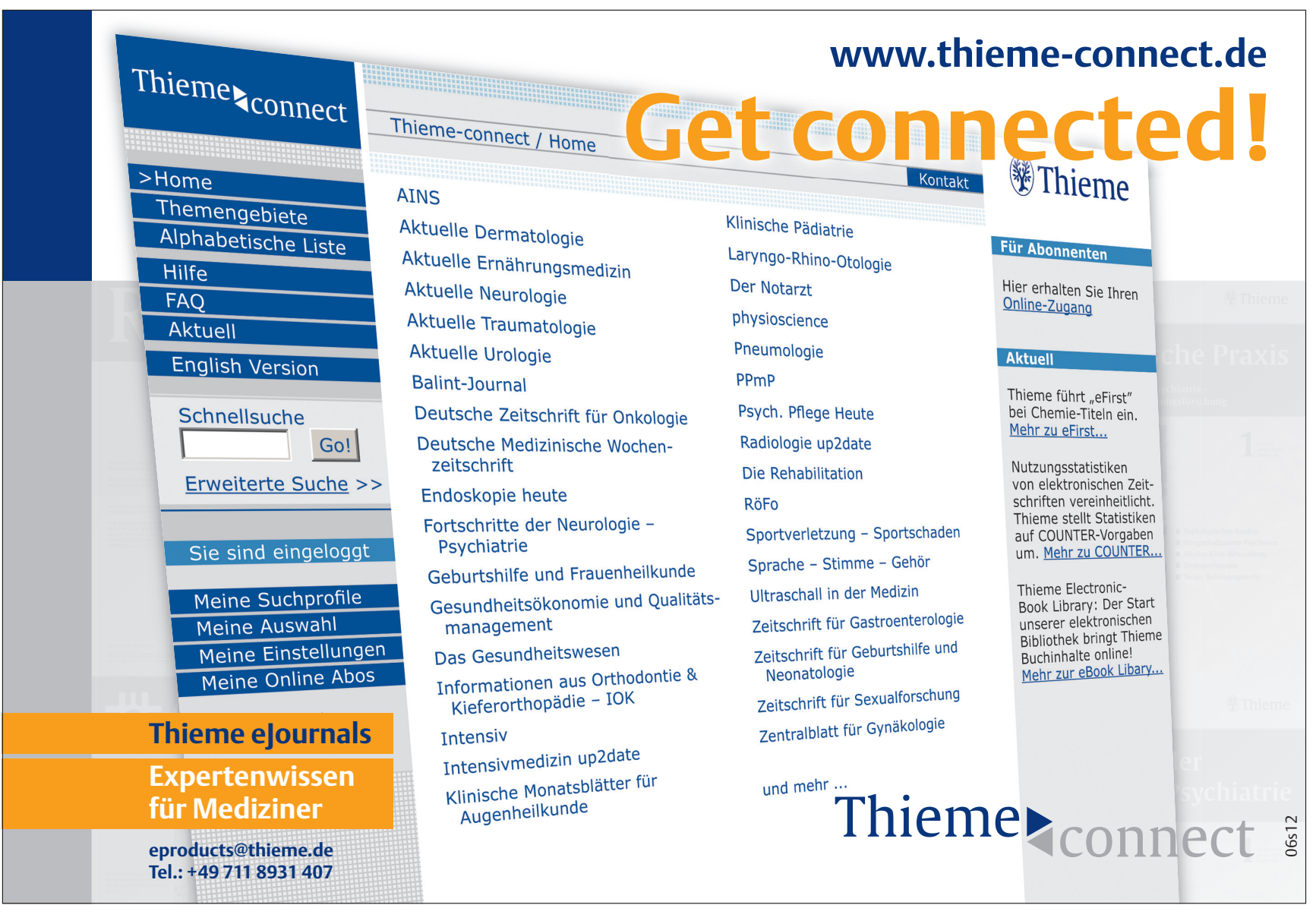




\section{„Ein Glasionomer mit überlegener Frakturresistenz in der Kausimulation“}

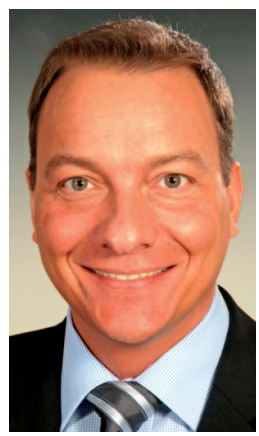

R. Frankenberger
In der restaurativen

Zahnheilkunde stehen

seit Langem konventi-

onelle Glasionomer-Zemente (GIZ) als Amalgam-Alternative zur Verfügung. Allerdings eignen sie sich mangels mechanischer Festigkeit nur für Provisorien oder semipermanente Füllungen. Eine neue Ära in der GlasionomerTechnologie leitet dagegen das neue ChemFil Rock (DENTSPLY DeTrey) ein. Es ermöglicht die Versorgung von Patienten, welche eine zuzahlungsfreie und dabei relativ langlebige Füllung wünschen. Über die Eigenschaften von ChemFil Rock und zur klinischen Bedeutung dieses erstmals zinkmodifizierten Glasionomers äußert sich Prof. Roland Frankenberger, Universität Marburg, im Interview mit Dentalfachjournalist Gerhard Frensel.
? Herr Prof. Frankenberger, welche Bedeutung haben heute GlasionomerZemente in der restaurativen Zahnheilkunde?

Prof. Roland Frankenberger: Aufgrund werkstoffkundlicher Überprüfungen sollte man erwarten, dass sie überhaupt keine Rolle in der zahnärztlichen Praxis spielten. Ihre Biegefestigkeit und Abrasionsstabilität reichen in der Regel nicht aus, um belastbare Füllungen herstellen zu können. Aber die Verkaufszahlen von Glasionomer-Zementen sprechen eine ganz andere Sprache. In nahezu jeder Zahnarztpraxis werden sie eingesetzt. GIZ sind beispielsweise ein probates Material für die temporäre Versorgung, oder wenn sich der Behandler nicht sofort auf die weitere Therapie festlegen beziehungsweise erst noch eine weitere Beratung des Patienten durchführen möchte. Glasionomer-Zemente bieten daher einen guten Weg, um mit dem Patienten „klar zu kommen“.

? Ist die Eigenschaft der Fluoridabgabe ein generelles Argument für GIZ?

Frankenberger: Diese ist sicherlich wünschenswert und mag gerade in der Alterszahnheilkunde eine Rolle spielen. Allerdings gibt es nur wenige wissenschaftliche Studien, die dezidiert nachweisen, dass die Fluoridabgabe wirklich dazu beiträgt, Sekundärkaries zu verhindern oder eine Füllung langlebiger zu gestalten.

? Inwieweit unterscheidet sich ChemFil Rock von konventionellen Glasionomer-Zementen?

Frankenberger: ChemFil Rock besitzt eine neuartige und intelligentere Chemie mit zinkmodifizierten Glasfüllern. Sie zielt vor allem darauf ab, dass das Material schneller als ein konventioneller GIZ mechanische Festigkeit entwickelt. Dadurch wird das Hauptproblem bisheriger Glasionomer-Zemente reduziert, welche während der frühen Phase - also etwa im Bereich der ersten Woche nach dem Legen der Füllung - leicht abradieren und sehr anfällig für Frakturen und Imperfektionen sind. Durch seine veränderte Che- mie erhält ChemFil Rock eine verbesserte Frakturresistenz und Abrasionsbeständigkeit - das haben wir in unserer Kausimulationsstudie nachgewiesen.

Bietet ChemFil Rock auch Vorteile bei der Verarbeitung?

Frankenberger: ChemFil Rock besitzt eine angenehme, nicht klebrige Konsistenz, die gut zu modellieren und leicht zu stopfen ist. Es adaptiert ausgezeichnet an die Kavitätenwände. Hier ist dem Hersteller ein vernünftiger Kompromiss gelungen. Die Verarbeitungsgeschwindigkeit ist sensationell, weil auf sämtliche Vorund Nachbehandlungen verzichtet werden kann. ChemFil Rock erfordert weder Konditionieren, noch Oberflächenversiegelung - ein großer Vorteil.

? Für welche Indikationen setzen Sie das Material ein?

Frankenberger: In erster Linie als Langzeitprovisorium. In meine Klinik kommen Patienten, die ChemFil Rock als AmalgamErsatz wünschen, eher selten. Allerdings warte ich mit Interesse auf die Ergebnisse von derzeit laufenden klinischen Studien zu diesem Thema. Es wird aktuell an der Ludwig-Maximilians-Universität München eine große Anwender-Feldstudie in Zahnarztpraxen durchgeführt, die anhand einer großen Fallzahl von ChemFil RockFüllungen dessen Eignung als AmalgamErsatz analysieren wird. Sollte die Studie signifikant belegen, dass nach 2 Jahren noch mehr als 95\% der ChemFil-Rock-Füllungen intakt sind, dann wäre das Material auch hinsichtlich der ADA-Kriterien für uns interessant.

Bei welchen Kavitäten-Klassen verwenden Sie ChemFil Rock?

Frankenberger: Bei Klasse-I- und mittelgroßen Klasse-II-Füllungen. Bei letzteren gehäuft, weil hier die Entscheidung darüber, ob direkt oder indirekt restauriert werden sollte, oftmals eine Übergangslösung mit einem GIZ erfordert.

Für welche Patientengruppen ist das Material besonders geeignet? 
Frankenberger: In erster Linie für den klassischen Nichtzuzahler, der ansonsten von der GKV nur die Versorgung mit Amalgam bezahlt bekäme. Dass - bei entsprechendem finanziellem Engagement des Patienten - eine Versorgung mit Komposit hochwertiger und langlebiger ist, steht natürlich außer Frage. Die Fähigkeit der Fluoridabgabe von ChemFil Rock könnte möglicherweise bei der Behandlung älterer Patienten vorteilhaft sein; aber bisher liegen hierzu kaum klinische Daten vor.

? Die Verarbeitung von ChemFil Rock geschieht im Vergleich zu anderen, auch teureren Materialien, besonders schnell. Wäre dies nicht ein Vorteil bei der Behandlung von Kindern?

Frankenberger: Grundsätzlich ja. Allerdings müssen wir auch hier noch die Ergebnisse einiger externer klinischer Studien abwarten, um die Eignung von ChemFil Rock für diesen Bereich genauer einschätzen zu können.

? Sie erwähnten aktuelle, derzeit laufende klinische Studien. In Ihrem eigenen Haus fanden umfangreiche InVitro-Testungen von ChemFil Rock und anderen Glasionomer-Zementen statt. Was genau wurde untersucht und welche Ergebnisse liegen Ihnen vor?

Frankenberger: Wir haben praktisch alle relevanten Parameter in vitro überprüft. ChemFil Rock wurde dabei komplett getestet und hat im Vergleich zu anderen GIZ sehr gut abgeschnitten. So wurde das Prüfmaterial in einer Kausimulation sowohl mechanisch als auch thermisch belastet: mit 100000 Kauzyklen und 2500 Thermozyklen bei $5^{\circ}$ und $55^{\circ} \mathrm{C}$. Im Gegensatz zu den konventionellen GIZ fanden wir bei ChemFil Rock keine Frakturen. Diese In-Vitro-Daten unserer Kausimulationsstudie entsprechen in etwa einer Tragedauer von 2 Jahren, wobei eine klinische Studie durch nichts zu ersetzen ist

? Was können Sie anhand der Datenlage bei ChemFil Rock Ihren Kollegen empfehlen?

Frankenberger: Wir dürfen uns nicht nur auf In-vitro-Daten stützen, sondern müssen noch auf aussagekräftige klinische
Daten warten, welche eine Tauglichkeit für dauerhaft verlässliche Seitenzahnrestaurationen belegen können. Deshalb empfehle ich derzeit, das neue Material für semipermanente Füllungen $\mathrm{zu}$ verwenden - mit Potenzial für mehr, wenn sich dies klinisch bestätigt. Positiv zu erwähnen ist noch, dass es bisher keine Unverträglichkeiten gegenüber diesem Material gegeben hat.

Welches vorläufige Fazit ziehen Sie zu ChemFil Rock?

Frankenberger: Es ist eine sehr schöne Neuentwicklung auf dem eigentlich alten Markt der Glasionomer-Zemente. Die bisherigen guten Erfahrungen mit ChemFil Rock machen neugierig auf mehr.

Korrespondenzadresse

Dentalfachjournalist Gerhard Frensel

Mentzhauser Straße 67

26939 Ovelgönne

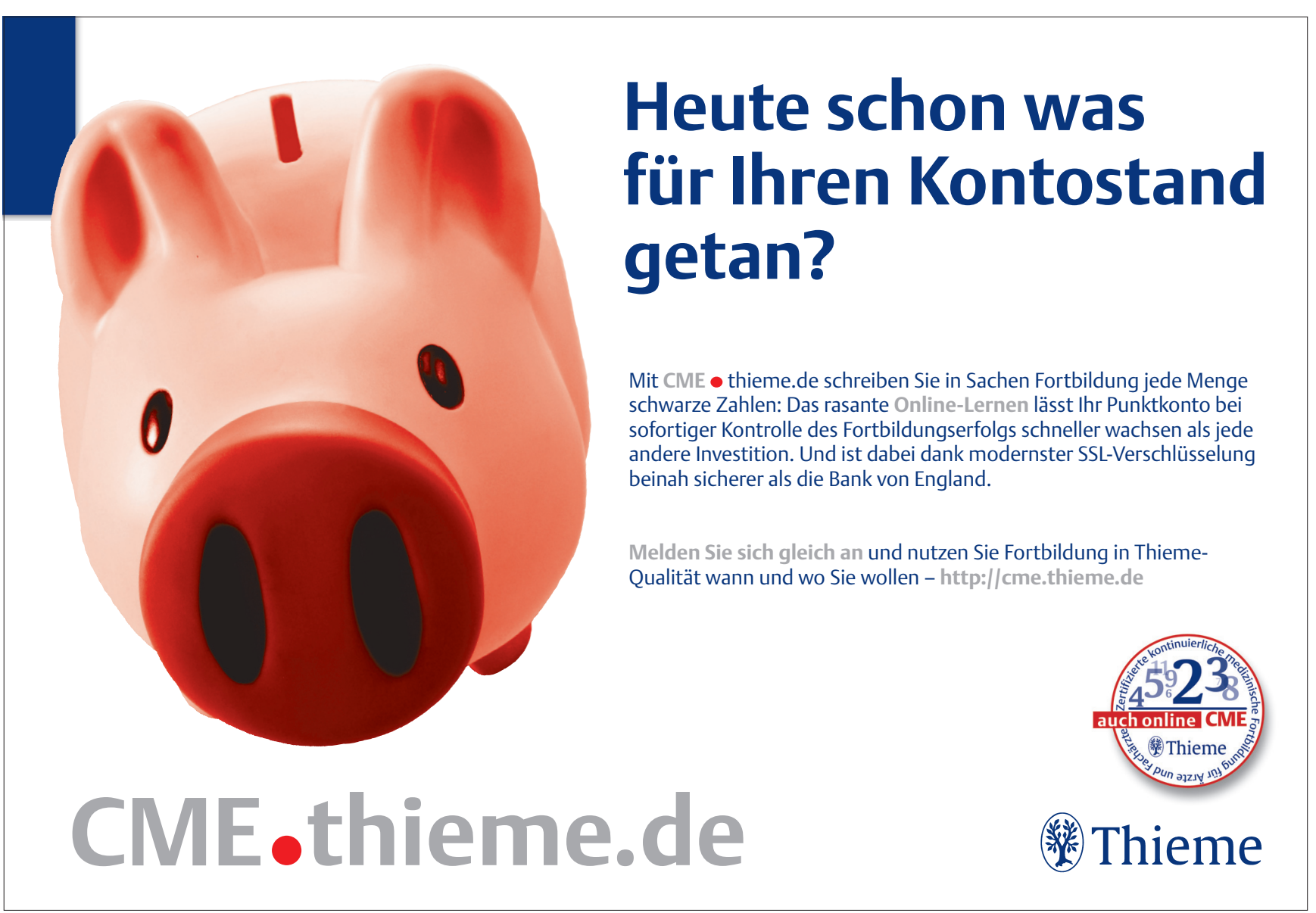




\section{„Ein CAD/CAM-System wie ARCTICA wird vom Markt erwartet"}

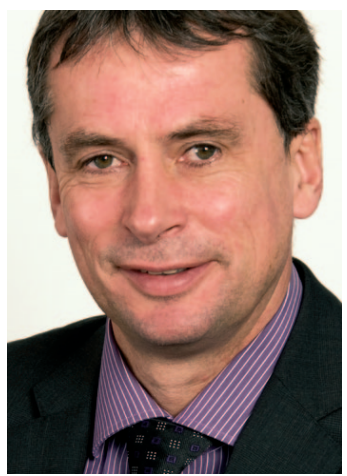

Henner Witte

\author{
Auf der IDS präsentierte \\ KaVo Dental das Future Concept \\ eines kompakten \\ Inhouse-CAD/CAM-Systems. \\ Jetzt kommt es auf den Markt.
}

\section{? Herr Witte, Sie versprechen den ARCTICA-} Käufern Investitionssicherheit. Wie sicher sind Sie, dass sich die Investitionen Ihres Unternehmens in die Entwicklung des ARCTICA CAD/ CAM-Systems auch rechnen?

Henner Witte: Sehr sicher. Ein CAD/ CAM-System wie ARCTICA wird erwartet - von den Zahnärzten, von den Laboren und dem Handel. Das hat uns die Projektphase „Future Concept“ gezeigt. Auf der IDS wurde das ARCTICA-Konzept den Fachkreisen präsentiert. Wir haben dort Meinungen gesammelt, diese verdichtet und in das Produkt einfließen lassen.

? Wie beschreiben Sie den typischen ARCTICA-Käufer?

Witte: Das werden Praxen und Zahntechniklabore sein, die inhouse eine kompakte CAD/CAM-Anlage haben möchten. Oder Praxen, die sogar ein eigenes Praxislabor haben und ihren Patienten modernste Zahnheilkunde bieten wollen.

Was sind die 3 wichtigsten Eigenschaften und Fähigkeiten, mit denen ARCTICA CAD/CAM punktet?

Witte: Vorneweg ist es die Wirtschaftlichkeit und auch die Amortisation der Investition. Wir bieten bei den Kosten Transparenz und Kalkulierbarkeit. Unsere kompakte 5-Achs-Nassfräs- und Schleifmaschine kann die geometrisch anspruchsvollsten Restaurationen präzise bearbeiten. Und wir haben gelernt, dass der Markt offene Systeme will. ARCTICA CAD/CAM kann diese Erwartung erfüllen. Qualitätsbracht?

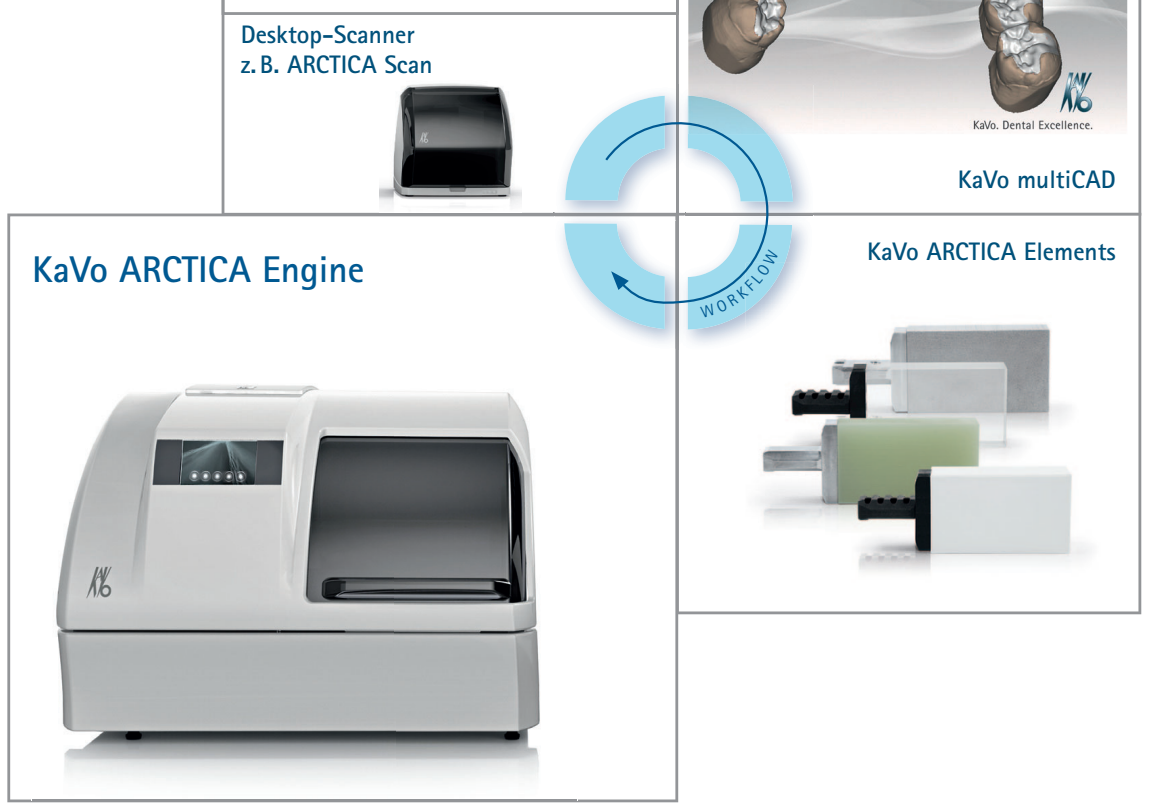

anbieter von Blöcken werden zukünftig ihre Materialien einbringen können, die den Qualitätsanforderungen von KaVo genügen. Und offene Schnittstellen erlauben den Upload offener STL-Daten unterschiedlicher am Markt befindlicher Scanner - auch von Intraoralscannern - in die KaVo multiCAD-Software, mit der sämtliche Restaurationen einfach und schnell konstruiert werden können.

Warum hat KaVo nicht selbst einen Intraoralscanner in das System einge-

Witte: Bei diesen Geräten gibt es immer noch wertvolle technologische Weiterentwicklungen. Unser Anspruch ist die Investitionssicherheit und Langlebigkeit. Und die sehen wir bei den Intraoralscannern noch nicht gegeben. Wir mauern hier nicht, sondern öffnen unser System all denen, die bereits heute solch ein Gerät besitzen.

Für die Praxen sind intraorale Scanner das Modul mit den dringendsten Verbesserungswünschen. Noch seien sie für viele
Patientenmünder zu voluminös und für die Hände der mitarbeitenden Damen meist viel zu schwer.

KaVo wird zu gegebener Zeit einen ausgereiften Intraoralscanner auf den Markt bringen.

? Aus welchen Komponenten besteht ARCTICA CAD/CAM? Was wird nun zur Markteinführung angeboten?

Witte: Das ARCTICA-System besteht aus 4 Komponenten: der ARCTICA Engine, dem ARCTICA Scan, der KaVo multiCAD-Software sowie einer Auswahl aller gängigen Materialkomponenten - von Titan über Zirkonoxid und Glaskeramik bis hin zu Kunststoffen und Wachs.

Dieses modulare System wird nun auf in Kürze in den Markt eingeführt.

\section{Herzlichen Dank, Herr Witte.}

Das Interview führte für die ZWR

Dr. Andreas Bachmann. den Fachdental-Messen präsentiert und 